\title{
Research institute partnerships 2015
}

\author{
Brady Huggett
}

With 11 deals, the US National Institutes of Health once again tops our list of industry partnering in 2015. Second on the list is Memorial Sloan Kettering, with five deals, the same number as 2014. Outside the United States, 2015 was a quiet year for the UK's Medical Research Council, which sealed just two deals (down from 11 in 2014; Fig. 1); the Paoli-Calmettes Institute in France and the Shanghai Cancer Institute in China entered the list for the first time. As always, oncology deals continue to dominate the landscape.
Table 1 Research institute partnerships 2015 Institute Partners

National Institutes of Health National Cancer Institute (NCI), SeraCare (11 deals)

Memorial Sloan Kettering (5 deals)

$\mathrm{NCl}$, Foundation Medicine

$\mathrm{NCl}$, Kite Pharma

$\mathrm{NCl}$, Lion Biotechnologies

National Center for Advancing Translational Sciences, Firststring

National Institutes of Health (NIH)

GlaxoSmithKline

$\mathrm{NIH}$, Wyeth Nutrition

NIH, GenVec

NIH, others

NIH, University of Massachusetts Medical School and Scripps, Padlock

NIH/National Institute of Alcohol Abuse and Alcoholism, Addex

Adimab

Genocea Biosciences

Cesca Therapeutics

Illumina

Peregrine Pharmaceuticals

Multiple Myeloma Research Inflection Biosciences

Foundation (3 deals)

GNS Healthcare

Adaptive, Onyx

Cold Spring Harbor (2 deals) Hairpin Technologies GlaxoSmithKline

\section{Details}

Cooperative Research and Development Agreement (CRADA) to create reference materials and positive controls for cancer assays.

Study on unique molecular indicators of tumors associated with exceptional drug responses.

Partners amend deal to include research on the immune response to tumor neoantigens. CRADA amended to include four new indications for tumor-infiltrating lymphocyte therapy.

CRADA to develop a connexin-based peptide therapeutic, ACT1, as a topical ophthalmic therapy for diabetic keratopathy and persistent corneal defects.

Partnership to develop one or more broadly neutralizing monoclonal antibodies against HIV infection.

Five-year partnership called the 'Baby Connectome Project' studying brains of healthy children up to 5 years old. Collaboration using GenVec's gorilla adenovirus vectors of low human seroprevalence to deliver antigens for a variety of malaria vaccine candidates.

Partners in an 'Alzheimer's Big Data' portal for use by the research community.

Collaboration to determine the role of individual peptidylarginine deiminase (PAD) enzymes in certain animal disease models and to evaluate PAD inhibitors in enzymatic assays, animal models and human cellular systems.

Collaboration to evaluate the pharmacology of ADX71441, a $\gamma$-aminobutyric acid $B$ receptor-positive allosteric modulator, in preclinical models of alcohol use disorder.

Collaboration with MD Anderson and Memorial Sloan Kettering on antibody discovery.

Collaboration to screen T-cell responses of melanoma and non-small-cell lung cancer patients treated with checkpoint inhibitors against patient-specific putative neoantigens.

Partnership to use Cesca's CellWerks portfolio of devices for intralaboratory stem cell purification applications.

Collaboration on noninvasive cancer diagnosis and monitoring.

Collaboration on combinations of phosphatidyl serine-targeting agents, including bavituximab, with other immune modulators.

Preclinical testing of Inflection's dual-mechanism phosphatidyl inositol mannoside/phosphatidyl inositol 3-kinase inhibitor, IBL-202.

Initiative to deploy predictive big data analytics on patient data from CoMMpass Study in multiple myeloma.

Collaboration to perform sequencing-based assessment of minimal residual disease on multiple myeloma patients over 5 years.

Initiative to expand the commercial distribution and research use of short hairpin RNA technology.

Collaboration with GSK on obesity and type 2 diabetes.

Collaboration to apply whole-genome, cancer and microbiome sequencing to heart disease.

Cleveland HeartLab to collaborate on a diagnostic and management solution for a heart disease biomarker discovered by the Cleveland Clinic.

Initiative to conduct translational research aimed at hematological cancers and at identifying associated biomarkers.

Collaboration to study protein-protein inhibitors for oncology indications.

Paoli-Calmettes Institute Innate Pharma

(2 deals)

Eisa

\section{First Rounders Podcast: Tom Maniatis}

Tom Maniatis is a founder of Kallyope, and head of the Maniatis lab at Columbia University. Among other things, he's founded several biotechs and authored a manual nicknamed "the bible of cloning." His conversation with Nature Biotechnology covers being the first in his family to go to college, the moratorium on recombinant DNA research in Cambridge, Massachusetts, and his long relationship with Jim Watson at Cold Spring Harbor. http://www.nature.com/nbt/podcast/index.html

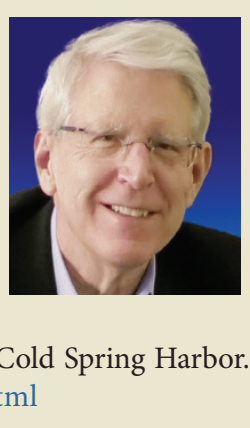

Figure 1 Most active research institutes, 2015. Source: BioCentury. 